\title{
POINCARÉ SERIES FOR PLANE CURVE SINGULARITIES AND THEIR BEHAVIOUR UNDER PROJECTIONS
}

\author{
JULIO JOSÉ MOYANO-FERNÁNDEZ
}

\begin{abstract}
Our purpose is to investigate all defined Poincaré series associated with multi-index filtrations and value semigroups of curve singularities - not necessarily complex - with regard to the property of forgetting variables, i.e., by making variables of the series to be 1. Generalised Poincaré series of motivic nature will be also considered.
\end{abstract}

\section{INTRODUCTION}

The idea of associating a generating function to a ring as a form of characterising properties of the ring goes back to R. Dedekind, as he was able to assimilate Riemann's philosophy in order to define the socalled Dedekind zeta functions for the ring of integers of a number field. The key point was to find a suitable way to measure filtrations of ideals (namely, the norm of an ideal in the ring of integers, since they are all finite). D. Hilbert and E. Noether extended later this argument to commutative algebra by introducing the Hilbert-Poincaré series of a module. As soon as the algebraic Geometry ripped in the shade of the fast development of the abstract algebra and the old numbertheoretical ideas, similar series were associated to affine rings of algebraic curves, giving rise to zeta functions of curves. A last step - much more recent and less explored - was to deal with singular algebraic curves over finite fields (cf. [14, [16, [22]). All mentioned zeta functions and Hilbert-Poincaré series can be understood as formal power

1991 Mathematics Subject Classification. Primary 14H20; Secondary 32S10, $11 \mathrm{G} 20$.

Key words and phrases. Curve singularity, Alexander polynomial, Poincaré series, value semigroup, Grothendieck ring of varieties, motivic integration.

The author was supported by the Deutsche Forschungsgemeinschaft (DFG), and partially by the Spanish Government grant "Ministerio de Educación y Ciencia (MEC) MTM2007-64704", in cooperation with the European Union in the framework of the founds "FEDER". 
series in one indeterminate. For the case of reduced curve singularities first, and in particular for complex plane curve singularities later, Campillo, Delgado, Kiyek and Gusein-Zade realised that a definition of multivariable Poincaré series (i.e. with several indeterminates, one for each irreducible component of the curve) not only makes sense but also yields a finer invariant of the singularity (see [10] and [3]-[7]).

The topological meaning of changing from one to more variables (for instance by making some variable to take the value 1) and back was cleared when Campillo, Delgado and Gusein-Zade showed the surprising connection between their Poincaré series and the Alexander polynomial for plane complex curve singularities (see [5]), because for this topological invariant, an old paper of Torres had already solved the problem [24, Theorem 3]. (Torres describes even its functional equations, see op.cit. Theorem 2).

Nevertheless Poincaré series are combinatorial objects definable not only in complex contexts. Our aim in this work is to understand the combinatorics behind the mechanism of passing from one to several variables. We understand that it may be useful, especially after a theorem of Delgado and the author saying that the information in the zeta functions of singular curves over finite fields - objects of numbertheoretical nature - is already contained in a generalised Poincaré series in the framework of motivic integration (by specialising in the cardinality of the finite field), establishing an interesting bridge between two a priori different objects (see [13]). Some related results in the flavour of that theorem were proven later on (e.g. [20], [15], [23], [21]).

We will keep the following notation throughout the paper. Set $I:=$ $\{1, \ldots, r\}$. For $i \in I$, we will write $\underline{1}_{i}$ for the $i$-th vector of the canonical basis of $\mathbb{Z}^{r}$, and $\underline{1}_{J}:=\sum_{j \in J} \underline{1}_{i}$ for every $J \subseteq I$. Notice that $\underline{1}_{I}=\underline{1}:=$ $(1,1, \ldots, 1)$ and $\underline{1}_{\varnothing}=\underline{0}:=(0,0, \ldots, 0)$.

\section{VAlue AND EXTENDED SEMigRoup}

Let $\mathcal{O}$ be a one-dimensional Cohen-Macaulay Noetherian local ring containing a perfect field $k$, with maximal ideal $\mathfrak{m}$. Let $\mathcal{K}$ be the total ring of fractions of $\mathcal{O}$ and let $\overline{\mathcal{O}}$ be the integral closure of $\mathcal{O}$ in $\mathcal{K}$. Assume the degree $\rho:=[\mathcal{O} / \mathfrak{m}: k]$ to be finite. By hypothesis, $\overline{\mathcal{O}}$ is an $\mathcal{O}$-module of finite length.

The integral closure $\overline{\mathcal{O}}$ decomposes as finite intersection of discrete Manis valuation rings (see [18]): $\overline{\mathcal{O}}=V_{1} \cap \ldots \cap V_{r}$, with associated

discrete Manis valuations $v_{1}, \ldots, v_{r}$. For every $i \in I$, we will write 
$\mathfrak{m}_{i}:=\mathfrak{m}\left(V_{i}\right) \cap \overline{\mathcal{O}}$, where $\mathfrak{m}\left(V_{i}\right)$ is the maximal ideal of $V_{i}$, and the residue fields will be denoted by $k_{i}:=V_{i} / \mathfrak{m}_{i}$.

We will assume that

$$
\mathcal{O} / \mathfrak{m}=\overline{\mathcal{O}} / \mathfrak{m}_{i}=V_{i} / \mathfrak{m}\left(V_{i}\right) \text { for every } i \in I .
$$

This hypothesis implies in particular that $\rho=\left[k_{i}: \mathcal{O} / \mathfrak{m}\right]=1$ for every $i \in I$, and we will refer to this property by saying that the ring $\mathcal{O}$ is residually rational (cf. [10, (4.1)]).

Thus, given such a ring $\mathcal{O}$ one can associate the set

$$
S=S_{\mathcal{O}}:=\left\{\underline{v}(z) \mid z \in \mathcal{O} \text { with } v_{i}(z)<\infty \text { for all } i \in I\right\}
$$

which is an additive sub-semigroup of $\mathbb{Z}_{\geq 0}^{r}$ : it is said to be the value semigroup associated to the ring $\mathcal{O}$. Notice that the semigroup $S$ possesses a conductor, say $\delta \in S$, i.e., the smallest element $\underline{v} \in S$ such that $\underline{v}+\mathbb{Z}_{\geq 0}^{r} \subseteq S$.

Let $\underline{v} \in \mathbb{Z}$, and $J \subseteq I$. Let us define the following subsets of the value semigroup $S$ :

$$
\begin{aligned}
\bar{\Delta}^{J}(\underline{v}) & :=\left\{\underline{w} \in \mathbb{N}^{r} \mid w_{i}>v_{i} \text { if } i \in J \text { and } w_{i}=v_{i} \text { if } i \notin J\right\} . \\
\Delta^{J}(\underline{v}) & :=\bar{\Delta}^{J}(\underline{v}) \cap S . \\
\Delta_{J}(\underline{v}) & :=\Delta^{I \backslash J}(\underline{v}) . \\
\Delta(\underline{v}) & :=\bigcup_{i=1}^{r} \Delta^{i}(\underline{n}) .
\end{aligned}
$$

A special role will be played by the following elements of $S$ (cf. [11]):

Definition 2.1. An element $\underline{v} \in S$ is said to be a maximal (of $S$ ) if $\Delta(\underline{v})=\varnothing$. If, moreover, one has that $\Delta_{J}(\underline{v})=\varnothing$ for every $J \subsetneq I$, $J \neq \varnothing$, then the element will be called an absolute maximal of $S$. If $\underline{v}$ is maximal and if $\Delta_{J}(\underline{v}) \neq \varnothing$ for every $J \subseteq I$ such that $\sharp(J) \geq 2$, then $\underline{v}$ will be called a relative maximal of $S$.

Let $\mathcal{O}$ be assumed to have a coefficient field $K$. Let $\left\{V_{1}, \ldots, V_{r}\right\}$ be the set of pairwise different Manis valuation rings of $\mathcal{K}$ belonging to $\mathcal{O}$.

If $K_{i}$ is a coefficient field of $V_{i}$ and $t_{i}$ is an indeterminate over $K_{i}$, then one can identify $V_{i} \cong K_{i} \llbracket t_{i} \rrbracket$ and $v_{i}$ with the order function respect to $t_{i}$ in $K_{i} \llbracket t_{i} \rrbracket$ for every $i \in I$. Hence

$$
\mathcal{O} \subset K_{1} \llbracket t_{1} \rrbracket \cap \ldots \cap K_{r} \llbracket t_{r} \rrbracket=\overline{\mathcal{O}} .
$$


Since $V_{i}$ is a module of finite type over the ring $\mathcal{O}$, the field extensions $\mathcal{O} / \mathfrak{m} \hookrightarrow \overline{\mathcal{O}} / \mathfrak{m}_{i}$ are finite, for every $i \in I$. Furthermore, as $\mathcal{O} / \mathfrak{m}$ is assumed to be perfect, every such a extension is separable and therefore, for every coefficient field $K$ of $\mathcal{O}$ there exists a unique coefficient field $K_{i}$ of $V_{i}$ with $K \subset K_{i}$ which is isomorphic to $\overline{\mathcal{O}} / \mathfrak{m}_{i}$ for every $i \in I$.

Define the ideals

$$
J(\underline{v}):=\{g \in \mathcal{O} \mid \underline{v}(g) \geq \underline{v}\},
$$

where $\underline{v}(g)=\left(v_{1}(g), \ldots, v_{r}(g)\right)$ and $\underline{v}(g) \geq \underline{v}$ means $v_{i}(g) \geq v_{i}$ for every $i \in I$. For every $\underline{v} \in \mathbb{Z}^{r}$ and every $i \in I$, let us consider the $\mathcal{O}$-module defined as

$$
C(\underline{v}, i):=J(\underline{v}) / J\left(\underline{v}+\underline{1}_{i}\right) .
$$

Notice that the $\mathcal{O}$-module $C(\underline{v}, i)$ is annihilated by $\mathfrak{m}$ so that $C(\underline{v}, i)$ naturally gets a structure of $k$-vector space.

Let us consider the map

$$
\begin{array}{ccc}
j_{\underline{v}}: \quad J(\underline{v}) & \longrightarrow C(\underline{v}, 1) \times \ldots \times C(\underline{v}, r) \\
g & \mapsto\left(j_{1}(g), \ldots, j_{r}(g)\right)=: j_{\underline{v}}(g) .
\end{array}
$$

We can identify $\operatorname{Im}\left(j_{\underline{v}}\right) \cong J(\underline{v}) / J(\underline{v}+\underline{1})=: C(\underline{v})$ and define the set

$$
F_{\underline{v}}:=C(\underline{v}) \cap((C(\underline{v}, 1) \backslash\{0\}) \times \ldots \times(C(\underline{v}, r) \backslash\{0\})) .
$$

\section{Lemma 2.2.}

$$
F_{\underline{v}}=C(\underline{v}) \cap\left(K_{1}^{*} \times \ldots \times K_{r}^{*}\right) .
$$

Proof. It is enough to see that the map $\varphi: C(\underline{v}, 1) \backslash\{0\} \rightarrow K_{1}^{*}$ is an isomorphism. Let be $g \in \mathcal{O} \backslash\{0\}$ with $v_{1}(g)=v_{1}$. One has that $g=a\left(t_{1}\right) t_{1}^{v_{1}(g)}+$ higher order terms, with $a\left(t_{1}\right) \in K_{1}^{*}$, thus $\varphi$ can be defined by $g \mapsto a\left(t_{1}\right)$.

In fact, if $\underline{v} \in \mathbb{Z}_{\geq 0}^{r}$ then one has

$$
F_{\underline{v}}=(J(\underline{v}) / J(\underline{v}+\underline{1})) \backslash \bigcup_{i=1}^{r}\left(J\left(\underline{v}+\underline{1}_{i}\right) / J(\underline{v}+\underline{1})\right),
$$

i.e., $F_{\underline{v}}$ is the complement to an arrangement of vector subspaces in a vector space (but not a vector subspace itself).

Definition 2.3. The extended semigroup $\widehat{S}$ is the union of the subspaces $F_{\underline{v}}$ for all $\underline{v} \in \mathbb{Z}_{\geq 0}^{r}$. The spaces $F_{\underline{v}}$ are called fibres of the extended semigroup $\widehat{S}$.

The group $K^{*}:=K \backslash 0$ acts freely on $\mathbb{Z}^{r} \times\left(K_{1}^{*} \times \ldots \times K_{r}^{*}\right)$ (by multiplication of all coordinates in $\left.K_{1}^{*} \times \ldots \times K_{r}^{*}\right)$. The corresponding factor space $\mathbb{Z}^{r} \times\left(K_{1}^{*} \times \ldots \times K_{r}^{*}\right) / K^{*}=\mathbb{Z}^{r} \times \mathbb{P}\left(K_{1}^{*} \times \ldots \times K_{r}^{*}\right)=$ $\sum_{\underline{v} \in \mathbb{Z}^{r}} \mathbb{P}\left(K_{1}^{*} \times \ldots \times K_{r}^{*}\right) \underline{t} \underline{\underline{v}}$ has the natural structure of semigroup. 
The extended semigroup $\widehat{S} \subset \mathbb{Z}^{r} \times\left(K_{1}^{*} \times \ldots \times K_{r}^{*}\right)$ is invariant with respect to the $K^{*}$-action. The factor space

$$
\mathbb{P} \widehat{S}=\widehat{S} / K^{*}
$$

is called the projectivisation of the extended semigroup (it is also a graded semigroup in a natural sense).

By the previous definitions, the projectivisation of the extended semigroup can be described as a sum

$$
\mathbb{P} \widehat{S}=\sum_{\underline{v} \in \mathbb{Z}^{r}} \mathbb{P} F_{\underline{v}} \cdot \underline{t}^{\underline{v}}
$$

where $\mathbb{P} F_{\underline{v}}=F_{\underline{v}} / K^{*}$ is the projectivisation of the fibre $F_{\underline{v}}$. For $\underline{v} \in \widehat{S}$, the space $\overline{\mathbb{P}} F_{\underline{v}}$ is the complement to an arrangement of projective hyperplanes in a $\left(\operatorname{dim}_{K}(J(\underline{v}) / J(\underline{v}+\underline{1}))-1\right)$-dimensional projective space $\mathbb{P}(J(\underline{v}) / J(\underline{v}+\underline{1}))$.

\section{The Poincaré Series associated With the Ring $\mathcal{O}$}

3.1. Multi-index filtrations and Poincaré series. Let us consider the multi-index filtration defined by the ideals $\{J(\underline{v})\}$, for a given vector $\underline{v}=\left(v_{1}, \ldots, v_{r}\right) \in \mathbb{Z}_{\geq 0}^{r}$. Since the $\operatorname{ring} \mathcal{O}$ is a one-dimensional reduced ring, it is Cohen-Macaulay, so the property of the ideals $J(\underline{v})$ of $\mathcal{O}$ of containing regular elements is equivalent to the property $\mathfrak{m}^{N} \supset J(\underline{v})$ for some integer $N$. This means that the filtration $\{J(\underline{v})\}$ is in this case finitely determined, and therefore every subspace $J(\underline{v})$ has finite codimension $\ell(\underline{v})$ in $\mathcal{O}$. In particular, the dimension

$$
c(\underline{v}):=\operatorname{dim}_{k}(J(\underline{v}) / J(\underline{v}+\underline{1}))
$$

is also finite, because of the relation

$$
\ell(\underline{v}+\underline{1})=\ell(\underline{v})+c(\underline{v}) .
$$

This multi-index filtration can also be described in terms of a multivariable Laurent series

$$
L\left(t_{1}, \ldots, t_{r}\right):=\sum_{\underline{v} \in \mathbb{Z}^{r}} c(\underline{v}) \cdot \underline{t}^{\underline{v}}
$$

where $\underline{t} \underline{v}:=t_{1}^{v_{1}} \cdot \ldots \cdot t_{r}^{v_{r}}$. We will also write $L(\underline{t})$ instead of $L\left(t_{1}, \ldots, t_{r}\right)$ if the number of variables is clear from the context. The series (2) is an element of the $\mathbb{Z}\left[t_{1}, \ldots, t_{r}\right]$-module (or even a module over the $\left.\operatorname{ring} \mathbb{Z}\left[t_{1}, \ldots, t_{r}, t_{1}^{-1}, \ldots, t_{r}^{-1}\right]\right) \mathbb{Z} \llbracket t_{1}, \ldots, t_{r}, t_{1}^{-1}, \ldots, t_{r}^{-1} \rrbracket$ (but not a ring, although the polynomial ring $\mathbb{Z}\left[t_{1}, \ldots, t_{r}\right]$ can be in a natural way considered to be embedded into it). That is, $L(\underline{t})$ is a Laurent series 
infinitely long in all directions (given by the vectors of the standard basis $\left.\left\{\underline{1}_{1}, \ldots, \underline{1}_{r}\right\}\right)$ since $c(\underline{v}):=\operatorname{dim}_{\mathbb{C}}(J(\underline{v}) / J(\underline{v}+\underline{1}))$ can be positive for $\underline{v}$ with (some) negative components $v_{i}$ as well. It is easy to see that, along each line in the lattice $\mathbb{Z}^{r}$ parallel to a coordinate one, the coefficients $c(\underline{v})$ stabilize in each direction; that is, if $v_{i}^{\prime}$ and $v_{i}^{\prime \prime}$ are negative, or if $v_{i}^{\prime}$ and $v_{i}^{\prime \prime}$ are positive and large enough, then $c\left(v_{1}, \ldots, v_{i}^{\prime}, \ldots v_{r}\right)=c\left(v_{1}, \ldots, v_{i}^{\prime \prime}, \ldots v_{r}\right)$. This implies that

$$
P^{\prime}\left(t_{1}, \ldots t_{r}\right)=\prod_{i=1}^{r}\left(t_{i}-1\right) L\left(t_{1}, \ldots, t_{r}\right)
$$

is a power series in $\underline{t}=t_{1} \cdot \ldots \cdot t_{r}(\mathrm{cf}$. [5]).

If we write $P^{\prime}\left(t_{1}, \ldots, t_{r}\right)=\sum_{\underline{v} \in \mathbb{Z}^{r}} p^{\prime}(\underline{v}) \underline{t} \underline{v}$, then for every $\underline{v} \in \mathbb{Z}^{r}$ we have

$$
\begin{aligned}
p^{\prime}(\underline{v}) & =(-1)^{r} \sum_{j=0}^{r}(-1)^{j} \sum_{1 \leq i_{1}<\ldots<i_{j} \leq r} c\left(\underline{v}-\underline{1}_{\left\{i_{1}, \ldots, i_{j}\right\}}\right) \\
& =(-1)^{r} \sum_{J \subseteq I}(-1)^{\sharp(J)} c\left(\underline{v}-\underline{1}_{J}\right) .
\end{aligned}
$$

Notice that $P^{\prime}\left(t_{1}, \ldots, t_{r}\right)$ is a polynomial in the indeterminates $t_{1}, \ldots, t_{r}$ with coefficients in $\mathbb{Z}$. Moreover, if $p^{\prime}(\underline{v}) \neq 0$, then $\underline{0} \leq \underline{v} \leq \delta$, where " $\leq$ " means the ordering given by the product.

Remark 3.1. The dimension $c(\underline{v})$ depends only on the value semigroup $S$. In fact, if $K \subseteq I$, let

$$
\varnothing \subsetneq K_{1} \subsetneq K_{2} \subsetneq \ldots \subsetneq K_{h-1} \subsetneq K_{h}=I
$$

be such that $\Delta^{K_{i}}(\underline{v}) \neq \varnothing$ and $h$ is maximal with these properties. If we choose $g_{i} \in J(\underline{v})$ such that $\underline{v}\left(g_{i}\right) \in \Delta^{K_{i}}(\underline{v})$ for $0 \leq i \leq h$, then $\left\{j_{\underline{v}}\left(g_{i}\right) \mid 0 \leq i \leq h-1\right\}$ is a basis of $C(\underline{v})$, therefore $h=c(\underline{v})$ (cf. [2]).

3.2. Computation of the coefficients of the Poincaré series. Let $\underline{v} \in \mathbb{Z}^{r}$ and $i \in I$. The vector space $C(\underline{v}, i)=J(\underline{v}) / J\left(\underline{v}+\underline{1}_{\{i\}}\right)$ can be identified with a vector subspace of the complex line. We will denote $c(\underline{v}, i):=\operatorname{dim}_{k} C(\underline{v}, i)$. It is clear that $c(\underline{v}, i)=1$ if and only if there exists $\underline{w} \in S$ such that $w_{i}=v_{i}$ and $w_{j} \geq v_{j}$ for every $j \in I$. For any reordering $\left\{i_{1}, \ldots, i_{r}\right\}=\{1, \ldots, r\}$ of the set $I$ one has

$$
C(\underline{v}) \cong \bigoplus_{j=0}^{r-1} C\left(\underline{v}+\underline{1}_{\left\{i_{1}, \ldots, i_{j}\right\}}, i_{j+1}\right)
$$


and therefore

$$
c(\underline{v})=\sum_{j=0}^{r-1} c\left(\underline{v}+\underline{1}_{\left\{i_{1}, \ldots, i_{j}\right\}}, i_{j+1}\right) .
$$

Let $\underline{v}, \underline{w} \in \mathbb{Z}^{r}$ and $\underline{v} \leq \underline{w}$; for every $i \in I$ there exists a canonical linear map $\varphi_{\underline{w}, \underline{v}, i}: C(\underline{w}, i) \rightarrow C(\underline{v}, i)$ induced by the inclusion $J(\underline{w}) \rightarrow J(\underline{v})$. If, moreover, $w_{i}=v_{i}$, then the map $\varphi_{\underline{w}, \underline{v}, i}$ is a monomorphism, i.e., $0 \leq c(\underline{w}, i) \leq c(\underline{v}, i) \leq 1$.

Let $\underline{v} \in \mathbb{Z}^{r}$ and fix $i \in I$. Define

$$
\begin{aligned}
p_{i}(\underline{v}):=(-1)^{r-1} \sum_{J \subseteq I \backslash\{i\}}(-1)^{\sharp(J)} c\left(\underline{v}+\underline{1}-\underline{1}_{\{i\}}-\underline{1}_{J}, i\right) \\
=(-1)^{r} \sum_{i \in J^{\prime} \subseteq I}(-1)^{\sharp\left(J^{\prime}\right)} c\left(\underline{v}+\underline{1}-\underline{1}_{J^{\prime}}, i\right)
\end{aligned}
$$

and let

$$
P_{i}\left(t_{1}, \ldots, t_{r}\right)=\sum_{\underline{v} \in \mathbb{Z}^{r}} p_{i}(\underline{v}) \underline{t} \underline{v} .
$$

The following result may be proved in much the same way as Proposition 8 in [12, p. 1649], we include however the proof by the sack of completeness.

Theorem 3.2. Let $\underline{v} \in \mathbb{Z}^{r}$ and $i \in I$. Then $p^{\prime}(\underline{v})=-p_{i}(\underline{v})+p_{i}(\underline{v}-\underline{1})$ and therefore

$$
\left(t_{1} \cdot \ldots \cdot t_{r}-1\right) \cdot P_{i}\left(t_{1}, \ldots, t_{r}\right)=P^{\prime}\left(t_{1}, \ldots, t_{r}\right) .
$$

As a consequence $P_{i}\left(t_{1}, \ldots, t_{r}\right)$ does not depend on $i$ (it will be denoted $P\left(t_{1}, \ldots, t_{r}\right)$ in the sequel).

Proof. The coefficient $p^{\prime}(\underline{v})$ can be written as

$$
p^{\prime}(\underline{v})=(-1)^{r} \sum_{i \notin J \subseteq I}(-1)^{\sharp(J)}\left(c\left(\underline{v}-\underline{1}_{J}\right)-c\left(\underline{v}-\underline{1}_{\{i\}}-\underline{1}_{J}\right)\right) .
$$

Let $\left\{i_{1}, \ldots, i_{r-1}\right\}$ be any subset of the set $I \backslash\{i\}$. Then one can compute each summand above as

$$
\begin{gathered}
c\left(\underline{v}-\underline{1}_{J}\right)=\sum_{j=0}^{r-2} c\left(\underline{v}-\underline{1}_{J}+\underline{1}_{\left\{i_{1}, \ldots, i_{j}\right\}}, i_{j+1}\right)+c\left(\underline{v}-\underline{1}_{J}+\underline{1}_{I \backslash\{i\}}, i\right) \\
c\left(\underline{v}-\underline{1}_{J}-\underline{1}_{\{i\}}\right)=c\left(\underline{v}-\underline{1}_{J}-\underline{1}_{\{i\}}, i\right)+\sum_{j=0}^{r-2} c\left(\underline{v}-\underline{1}_{J}+\underline{1}_{\left\{i_{1}, \ldots, i_{j}\right\}}, i_{j+1}\right)
\end{gathered}
$$


and as a consequence one has

$$
\begin{aligned}
p^{\prime}(\underline{v}) & =(-1)^{r} \sum_{i \notin J \subseteq I}(-1)^{\sharp(J)}\left(c\left(\underline{v}-\underline{1}_{J}-\underline{1}_{\{i\}}+\underline{1}_{i}, i\right)-c\left(\underline{v}-\underline{1}_{\{i\}}-\underline{1}_{J}, i\right)\right) \\
& =-p_{i}(\underline{v})+p_{i}(\underline{v}-\underline{1}) .
\end{aligned}
$$

Definition 3.3. The polynomial $P\left(t_{1}, \ldots, t_{r}\right)$ is called the Poincaré polynomial associated with $\mathcal{O}$.

The dimensions $c(\underline{v})$ satisfy the following symmetry property (cf. [10, Corollary 3.7]; remember that we assumed the $\operatorname{ring} \mathcal{O}$ to be residually rational):

Theorem 3.4 (Campillo, Delgado, Kiyek). Let $\underline{v} \in \mathbb{Z}^{r}$. Then

$$
c(\underline{v})+c(\delta-\underline{v}-\underline{1}) \leq r .
$$

Furthermore, the ring $\mathcal{O}$ is Gorenstein if and only if the equality holds.

The Poincaré polynomial associated with $\mathcal{O}$ satisfies the following functional equation if the ring $\mathcal{O}$ is assumed to be Gorenstein:

Theorem 3.5 (Campillo, Delgado, Kiyek). If $\mathcal{O}$ is Gorenstein, then we have

$$
P^{\prime}\left(t_{1}, \ldots, t_{r}\right)+(-1)^{r} \underline{t}^{\delta} P^{\prime}\left(t_{1}^{-1}, \ldots, t_{r}^{-1}\right)=0 .
$$

This formula gives immediately the relation

$$
P\left(t_{1}, \ldots, t_{r}\right)=(-1)^{r} \underline{t}^{\tau} P\left(t_{1}^{-1}, \ldots, t_{r}^{-1}\right),
$$

with $\tau:=\delta-\underline{1}$, hence the coefficients satisfy

$$
p(\underline{v})=(-1)^{r} p(\tau-\underline{v})
$$

for all $\underline{v} \in \mathbb{Z}_{\geq 0}^{r}$. Next lemma gives some computations on the coefficients $p(\underline{v})$ of the Poincaré series $P\left(t_{1}, \ldots, t_{r}\right)$ of $\mathcal{O}$.

Proposition 3.6. We have:

(1) If $\underline{v} \notin S$, then $p(\underline{v})=0$.

(2) If $\underline{v} \in S$ is not a maximal element of $S$, then $p(\underline{v})=0$.

(3) If $\underline{v}$ is an absolute maximal, then $p(\underline{v})=1$; if $\underline{v}$ is a relative maximal, then $p(\underline{v})=(-1)^{r}$.

Proof. We can fix any index $i \in I$ in order to compute $p(\underline{v})=p_{i}(\underline{v})$; then let us fix without loss of generality $i=r$. If $\left\{i_{1}, \ldots, i_{r-1}\right\}$ is any subset of $\{1, \ldots, r-1\}$, we have

$$
\begin{gathered}
0 \leq \quad c\left(\underline{v}+\underline{1}-\underline{1}_{\{r\}}, r\right) \leq c\left(\underline{v}+\underline{1}-\underline{1}_{\{r\}}-\underline{1}_{\left\{i_{1}\right\}}, r\right) \leq \ldots \\
\ldots \leq c\left(\underline{v}+\underline{1}-\underline{1}_{\{r\}}-\underline{1}_{\left\{i_{1}, \ldots, i_{r-1}\right\}}, r\right)=c(\underline{v}, r) \leq 1 .
\end{gathered}
$$


If $c\left(\underline{v}+\underline{1}-\underline{1}_{\{r\}}, r\right)=1$, then all terms involved in the expression

$$
p_{r}(\underline{v})=(-1)^{r-1} \sum_{j=0}^{r-1}(-1)^{j} \sum_{1 \leq i_{1}<\ldots<i_{j} \leq r-1} c\left(\underline{v}+\underline{1}-\underline{1}_{\left\{i_{1}, \ldots, i_{j}\right\}}, r\right)
$$

are equal to 1 . As a consequence we have

$$
p_{r}(\underline{v})=(-1)^{r-1} \sum_{j=0}^{r-1}(-1)^{j}\left(\begin{array}{c}
r-1 \\
j
\end{array}\right)=(-1)^{r-1}(1-1)^{r-1}=0 .
$$

If $c(\underline{v}, r)=0$, then all terms are equal to 0 and therefore $p_{r}(\underline{v})=0$. Now, let $\underline{v} \notin S$; then there exists $i \in\{1, \ldots, r\}$ such that $c(\underline{v}, i)=0$ and by the previous computations we have $p(\underline{v})=p_{i}(\underline{v})=0$. If $\underline{v} \in S$ is not maximal, then there exists an index $i$ such that $\Delta_{\{i\}}(\underline{v}) \neq \varnothing$, but this is equivalent to say $c\left(\underline{v}+\underline{1}-\underline{1}_{\{i\}}, i\right)=1$, and therefore $p(\underline{v})=$ $p_{i}(\underline{v})=0$. Let $\underline{v} \in S$ be an absolute maximal; then $\Delta_{K}(\underline{v}) \neq \varnothing$ for every $K \subset I$, and $c(\underline{v}, r)=1$ and $c\left(\underline{v}+\underline{1}_{-1} \underline{1}_{\{r\}}-\underline{1}_{J}, r\right)=0$ for every $J \subset I \backslash\{r\}$. Hence $p(\underline{v})=(-1)^{r-1}(-1)^{r-1}=1$. The condition for $\underline{v}$ to be a relative maximal is that $\Delta_{K}(\underline{v}) \neq \varnothing$ for every $K$ such that $\sharp(K) \geq 2$ (and it is empty if $\sharp(K)=1$ ). Therefore $c\left(\underline{v}+\underline{1}-\underline{1}_{\{r\}}, r\right)=0$ and $c\left(\underline{v}+\underline{1}-\underline{1}_{\{r\}}-\underline{1}_{J}, r\right)=1$ for every $\varnothing \neq J \subset I \backslash\{r\}$. Then we have

$$
p(\underline{v})=(-1)^{r-1} \sum_{j=1}^{r-1}(-1)^{j}\left(\begin{array}{c}
r-1 \\
j
\end{array}\right)=(-1)^{r} .
$$

Remark 3.7. From the above computations we get nice descriptions of $P\left(t_{1}, \ldots, t_{r}\right)$ for $r \in\{2,3\}$. Indeed, if $r=2$, there is no difference between relative and absolute maximals and we have

$$
P\left(t_{1}, t_{2}\right)=\sum_{\underline{v} \text { maximal }} \underline{t^{v}}
$$

If $r=3$, then we have only relative and absolute maximals and we obtain

$$
P\left(t_{1}, t_{2}, t_{3}\right)=-\sum_{\underline{v} \text { rel. max. }} \underline{t}^{\underline{v}}+\sum_{\underline{v} \text { abs. max. }} \underline{t}^{\underline{v}}
$$

\section{Two Possible DeFinitions AND ITS EQUiVALENCE}

The definition of Poincaré series for $\mathcal{O}$ that we have chosen as a "tentative" is not the only possible. First of all, if a vector $\underline{v} \in \mathbb{Z}^{r}$ belongs to $S$, then it seems to be natural to take at least the "dimension" of its fiber $F_{\underline{v}}$ in the extended semigroup as a way to measure (or "count") the elements $g \in \mathcal{O}$ with $\underline{v}(g)=\underline{v}$. However, $F_{\underline{v}}$ is not a vector subspace 
of $k^{r}$ but a linear subspace minus some hyperplanes, more precisely $F_{\underline{v}}=C(\underline{v}) \cap(k \backslash\{0\})$, also $\overline{F_{\underline{v}}}=C(\underline{v})$. As a consequence, any definition of Poincaré series must take as a coefficient of $\underline{t} \underline{v}$ at least the dimension of $C(\underline{v})$. Note that for any $\underline{v} \in \mathbb{N}^{r}$ (indeed for any $\underline{v} \in \mathbb{Z}^{r}$ with at least one coordinate greater that or equal to zero) the vector space $C(\underline{v})$ is different from 0 , even in the case $F_{\underline{v}}=\varnothing$ if $\underline{v} \notin S$.

Another natural definition for the Poincaré series is

$$
\widetilde{L}_{S}(\underline{t})=\sum_{\underline{v} \in \mathbb{N}^{r}} c(\underline{v}) \underline{t} \underline{v} \in \mathbb{Z} \llbracket t_{1}, \ldots, t_{r} \rrbracket .
$$

Using similar computations as those in the subsection 3.2 we can also take the polynomial

$$
\widetilde{P}_{S}(\underline{t})=\left(t_{1}-1\right) \cdot \ldots \cdot\left(t_{r}-1\right) \widetilde{L}_{S}(\underline{t})=\sum_{\underline{v} \in \mathbb{N}^{r}} \widetilde{p}(\underline{v}) \underline{t}^{\underline{v}} .
$$

For any $\underline{v}$ with $v_{i}>0$ for $i \in I$ it obviously holds $\widetilde{p}(\underline{v})=p^{\prime}(\underline{v})$, hence the differences between $\widetilde{P}$ and $P^{\prime}$ appear only in the intersections of $\mathbb{N}^{r}$ with the coordinate hyperplanes. More precisely, let $A \subseteq I$, and let $S_{A}$ be the semigroup arising from $S$ by projection on the set of indices $A$ (i.e. $S_{A}$ is the value semigroup associated with the curve consisting of the branches given by $A$ ). We have:

Theorem 4.1. If $r=1$, then $\widetilde{P}_{S}=P_{S}^{\prime}$. For $r \geq 2$ we have

$$
\widetilde{P}_{S}(\underline{t})=\sum_{\varnothing \neq A \subseteq I}(-1)^{\sharp(I \backslash A)} P_{S_{A}}^{\prime}\left(\underline{t}_{A}\right),
$$

where $\underline{t}_{A}:=\prod_{i \in A} t_{i}$. As a consequence, one has

$$
P_{S}^{\prime}(\underline{t})=\sum_{\varnothing \neq A \subseteq I}(-1)^{\sharp(I \backslash A)} \widetilde{P}_{S_{A}}\left(\underline{t}_{A}\right) .
$$

Proof. Take $J \subseteq I$, and consider $\Lambda_{J}(\underline{t}):=\sum_{\underline{v} \in \bar{\Delta}^{J}(\underline{0})} \tilde{p}(\underline{v}) \underline{t} \underline{v}$ so that

$$
\widetilde{P}_{S}(\underline{t})=\sum_{J \subseteq I} \Lambda_{J}\left(\underline{t}_{J}\right) .
$$

Let $J \neq \varnothing$. It is easily seen that

$$
\Lambda_{J}\left(\underline{t}_{J}\right)=(-1)^{\sharp(I \backslash J)}\left(P_{S_{J}}^{\prime}\left(\underline{t}_{J}\right)-p_{s_{J}}^{\prime}(\underline{v})\right) .
$$

We know that $\widetilde{p}_{S}(\underline{v})=p(\underline{v})=(-1)^{r}$ and that $c\left(\underline{v}-\underline{1}_{J}\right)=1$ for $J \subseteq I$, $J \neq I$, and $c\left(\underline{v}-\underline{1}_{J}\right)=0$ for $J=I$, therefore

$$
p_{S}^{\prime}(\underline{v})=p^{\prime}(\underline{v})=(-1)^{r} \sum_{J \subsetneq I}=(-1)^{r} \sum_{i=0}^{r-1}(-1)^{i}\left(\begin{array}{l}
r \\
i
\end{array}\right)=(-1)^{2 r-1}=-1,
$$


and so

$$
\Lambda_{J}\left(\underline{t}_{J}\right)=(-1)^{\sharp(I \backslash J)}\left(P_{S_{J}}^{\prime}\left(\underline{t}_{J}\right)+1\right) .
$$

We have

$$
\widetilde{P}_{S}(\underline{t})=\sum_{\varnothing \neq J \subseteq I} \Lambda_{J}\left(\underline{t}_{J}\right)+\Lambda_{\varnothing}\left(\underline{t}_{\varnothing}\right)
$$

where $\Lambda_{\varnothing}\left(\underline{t}_{\varnothing}\right)=\widetilde{p}(\underline{0})=(-1)^{r}$, hence we obtain:

$$
\begin{aligned}
\widetilde{P}_{S}(\underline{t}) & =\sum_{\varnothing \neq J \subseteq I}(-1)^{\sharp(I \backslash J)}\left(P_{S_{J}}^{\prime}\left(\underline{t}_{J}\right)-p_{s_{J}}^{\prime}(\underline{v})\right)+(-1)^{r} \\
& =\sum_{\varnothing \neq J \subseteq I}(-1)^{\sharp(I \backslash J)} P_{S_{J}}^{\prime}\left(\underline{t}_{J}\right)-\sum_{\varnothing \neq J \subseteq I}(-1)^{\sharp(I \backslash J)} p_{s_{J}}^{\prime}(\underline{v})+(-1)^{r} \\
& =\sum_{\varnothing \neq J \subseteq I}(-1)^{\sharp(I \backslash J)} P_{S_{J}}^{\prime}\left(\underline{t}_{J}\right)-\sum_{\varnothing \neq J \subseteq I}(-1)^{\sharp(I \backslash J)}(-1)+(-1)^{r} \\
& =\sum_{\varnothing \neq J \subseteq I}(-1)^{\sharp(I \backslash J)} P_{S_{J}}^{\prime}\left(\underline{t}_{J}\right)+\sum_{\varnothing \neq J \subseteq I}(-1)^{\sharp(I \backslash J)}+(-1)^{r} \\
& =\sum_{\varnothing \neq J \subseteq I}(-1)^{\sharp(I \backslash J)} P_{S_{J}}^{\prime}\left(\underline{t}_{J}\right)+\sum_{i=1}^{r}(-1)^{i}\left(\begin{array}{l}
r \\
i
\end{array}\right)+(-1)^{r} \\
& =\sum_{\varnothing \neq J \subseteq I}(-1)^{\sharp(I \backslash J)} P_{S_{J}}^{\prime}\left(\underline{t}_{J}\right)-(-1)^{r}+(-1)^{r} \\
& =\sum_{\varnothing \neq J \subseteq I}(-1)^{\sharp(I \backslash J)} P_{S_{J}}^{\prime}\left(\underline{t}_{J}\right),
\end{aligned}
$$

as desired.

Remark 4.2. Formulae above express the equivalence between the sets of polynomials $\left\{P_{S_{A}}^{\prime} \mid A \subseteq I\right\}$ and $\left\{\widetilde{P}_{S_{A}} \mid A \subseteq I\right\}$. In fact, $\widetilde{P}_{S_{A}}$ can be obtained from $\widetilde{P}_{S}$ just by substituting $t_{i}=0$ for any $i \notin A$ and multiplying by $(-1)^{r-\sharp(A)}$. Then the polynomial $\widetilde{P}_{S}$ is equivalent to the set of polynomials $\left\{P_{S_{A}}^{\prime} \mid A \subseteq I\right\}$. From this point of view there is no difference between the two definitions (see also Section 5).

For some purposes it is sometimes useful to consider codimensions of the ideals $J(\underline{v})$ as coefficients of the corresponding Poincaré series, what leads to a third definition:

$$
H(\underline{t}):=\sum_{\underline{v} \in \mathbb{N}^{r}} \ell(\underline{v}) \underline{t}^{v},
$$

where $\ell(\underline{v})=\operatorname{dim}(\mathcal{O} / J(\underline{v}))$ is the codimension of the ideal $J(\underline{v})$ in the ring $\mathcal{O}$ (cf. Subsection 3.1). As a consequence of Theorem 4.1 one can prove the following relation between the Poincaré series $H(\underline{t})$ and $P(\underline{t})$ : 
Corollary 4.3. We have

$$
\prod_{i=1}^{r}\left(1-t_{i}\right) H(\underline{t})=\sum_{\varnothing \neq A \subseteq I}(-1)^{\sharp(A)-1} \underline{t}_{A} P_{S_{A}}\left(\underline{t}_{A}\right) .
$$

Proof. Set $Q(\underline{t}):=\underline{t} \cdot P(\underline{t})$. It is enough to realise that

$$
Q(\underline{t})=(-1)^{r} \prod_{i=1}^{r}\left(1-t_{i}\right) H(\underline{t})
$$

(using the fact that $\ell(\underline{v}+\underline{1})=\ell(\underline{v})+c(\underline{v})$ for any $\underline{v} \in \mathbb{Z}^{r}$ ). By applying Theorem 4.1 to the series $Q(\underline{t})$, we are done.

\section{Behaviour BY PROJECTIONS}

In this section we want to analyse the behaviour by projections of the multi-variable Poincaré series, i.e., geometrically speaking, the behaviour of the Poincaré series of a reduced but not irreducible plane curve singularity by removing some of its branches. Consider

$$
J=\left\{i_{1}, \ldots, i_{h}\right\} \subset I=\{1, \ldots, r\} .
$$

Denoting by $\operatorname{pr}_{J}$ the projection over the indexes $J$ of $\mathbb{Z}_{+}^{r}$ in $\mathbb{Z}_{+}^{\sharp J}$. We set

$$
S_{\left\{i_{1}, \ldots, i_{h}\right\}}=S_{J}:=\operatorname{pr}_{J}(S)=\left\{\operatorname{pr}_{J}(\underline{v}) \mid \underline{v} \in S\right\} .
$$

That is, the semigroup $S_{J}$ corresponds to the projection of $S_{I}$ on the branches indexed by $J$.

Let $\xi_{i, j}$ denote the intersection multiplicity between the branches $C_{i}$ and $C_{j}$ given by the local rings $V_{i}$ and $V_{j}$, for every $i, j \in\{1, \ldots, r\}$ with $i \neq j$.

Theorem 5.1. Let $P_{S_{\{r\}}}\left(t_{1}, \ldots, t_{r-1}\right)$ be the Poincaré polynomial associated with the ring consisting of the branches $C_{1}, \ldots, C_{r-1}$ corresponding to the local rings $V_{1}, \ldots, V_{r-1}$, and $P\left(t_{1}, \ldots, t_{r}\right)$ the Poincaré polynomial of $\mathcal{O}$. We have

$$
P\left(t_{1}, \ldots, t_{r-1}, 1\right)=\left(1-t_{1}^{\xi_{1, r}} \cdot \ldots \cdot t_{r-1}^{\xi_{r-1, r}}\right) P_{S_{\{r\}}}\left(t_{1}, \ldots, t_{r-1}\right) .
$$

The same formula holds for $P^{\prime}$.

Proof. By the description of the Poincaré series $P_{S_{\{r\}}}^{\prime}(\underline{t})$ given by (4) we have

$$
\begin{aligned}
\left(1-\underline{t}^{\xi^{(r)}}\right) P_{S_{\{r\}}}^{\prime}\left(t_{1}, \ldots, t_{r-1}\right) & =\sum_{\underline{v} \in S_{\{r\}}} p_{S_{\{r\}}}^{\prime}(\underline{v}) \underline{t} \underline{v}-\sum_{\underline{v} \in S_{\{r\}}} p_{S_{\{r\}}}^{\prime}(\underline{v}) \underline{t}_{r} \underline{\underline{v}} \underline{\xi}_{r}^{(r)} \\
& =\sum_{\underline{v} \in S_{\{r\}}} A(\underline{v}) \underline{t} \underline{v}
\end{aligned}
$$


for $A(\underline{v}):=p_{S_{\{r\}}}^{\prime}(\underline{v})-p_{S_{\{r\}}}^{\prime}\left(\underline{v}-\xi^{(r)}\right)$ and with $\underline{t}_{r}:=t_{1}^{v_{1}} \cdot \ldots \cdot t_{r-1}^{v_{r-1}}$ and $\underline{t}_{r}^{\xi^{(r)}}:=t_{1}^{\xi_{1, r}} \cdot \ldots \cdot t_{r-1}^{\xi_{r-1, r}}$. The key point here is to understand the behaviour of the coefficients of the Poincaré series under semigroup projections. Let us present this for $S_{\{r\}}$, and the result follows by induction.

If we consider coefficients $p_{S_{\{r\}}}^{\prime}(\underline{w})$ in the fiber of such a semigroup projection of a fixed element $\underline{w}=\left(w_{1}, \ldots, w_{r-1}\right) \in S_{\{r\}}$, we realise first that the coefficients $p_{S_{\{r\}}}^{\prime}(\underline{w})$ of the projection series can be written as a finite sum $\sum_{\nu=0}^{u} p^{\prime}(\underline{w}, \nu)$. Indeed, our aim is to show the equality

$$
A(\underline{v})=\sum_{\nu=0}^{u} p^{\prime}(\underline{w}, \nu),
$$

and then we will be done. A first remarkable fact is the following: From the definitions of $P_{S}^{\prime}\left(t_{1}, \ldots, t_{r}\right)$ and $P_{S_{\{r\}}}^{\prime}\left(t_{1}, \ldots, t_{r-1}\right)$, the only remaining summands in the coefficients $p_{S}^{\prime}(\underline{v})$ are

$$
(-1)^{r} \sum_{r \notin J}(-1)^{\sharp(J)} c\left((\underline{w}, u)-\underline{1}_{J}\right)+(-1)^{r} \sum_{r \in J}(-1)^{\sharp(J)} c\left((\underline{w}, 1)-\underline{1}_{J}\right) .
$$

Next we apply the symmetry properties given by Theorem 3.4 to show

$$
c\left((\underline{w}, u)-\underline{1}_{J}\right)=r-c\left(\delta-\underline{1}-(\underline{w}, u)+\underline{1}_{J}\right),
$$

because our curve is assumed to be plane, hence Gorenstein. Denote $J^{\prime}:=I \backslash J$. Since $c\left((\underline{w}, u)-\underline{1}_{J}\right)=r-c\left((\delta-\underline{w}, 0)-\underline{1}_{J^{\prime}}\right)$, the term $(-1)^{r} \sum_{r \notin J}(-1)^{\sharp(J)} c\left((\underline{w}, u)-\underline{1}_{J}\right)$ is equal to

$$
\begin{aligned}
& (-1)^{r} \sum_{r \notin J}(-1)^{\sharp(J)}\left(r-c\left((\delta-\underline{v}, 0)-\underline{1}_{I \backslash J}\right)\right)= \\
= & (-1)^{r} \sum_{r \notin J}(-1)^{\sharp(J)}-(-1)^{r} \sum_{r \notin J}(-1)^{\sharp(J)} c\left((\delta-\underline{w}, 0)-\underline{1}_{J^{\prime}}\right)=(*)
\end{aligned}
$$

One may easily prove that $\sum_{r \notin J}(-1)^{\sharp(J)}=\sum_{i=0}^{r-1}(-1)^{i}\left(\begin{array}{c}r-1 \\ i\end{array}\right)=0$, hence

$$
\begin{aligned}
(*) & =(-1)^{r-1} \sum_{r \in J^{\prime}}(-1)^{r-\sharp\left(J^{\prime}\right)} c\left((\delta-\underline{w}, 0)-\underline{1}_{J^{\prime}}\right) \\
& =(-1)^{r}\left((-1)^{r-1} \sum_{r \in J^{\prime}}(-1)^{\sharp\left(J^{\prime}\right)} c\left((\delta-\underline{w}, 0)-\underline{1}_{J^{\prime}}\right)\right) .
\end{aligned}
$$

On the other hand, it is easily checked that

$$
(-1)^{r} \sum_{r \in J^{\prime}}(-1)^{\sharp\left(J^{\prime}\right)} c\left((\underline{w}, h)-\underline{1}_{J^{\prime}}\right)=p_{S_{\{r\}}}^{\prime}(\underline{w})
$$


for $h \in\{0,1\}$, and therefore

$$
\sum_{\nu=1}^{u} p^{\prime}(\underline{w}, \nu)=(-1)^{r-1} p_{S_{\{r\}}}^{\prime}(\delta-\underline{w})+p_{S_{\{r\}}}^{\prime}(\underline{w}) .
$$

By applying Theorem 3.5 to the coefficients $p_{S_{\{r\}}}^{\prime}(\underline{w})$ we get

$$
(-1)^{r-1} p_{S_{\{r\}}}^{\prime}(\delta-\underline{w})=-p_{S_{\{r\}}}^{\prime}\left(\underline{w}-\xi^{(r)}\right)
$$

(since $\left.\delta-\underline{w}=\delta\left(S_{\{r\}}\right)+\left(\xi_{1, r}, \ldots, \xi_{r-1, r}\right)-\underline{w}=\delta\left(S_{\{r\}}\right)-\left(\underline{w}-\xi^{(r)}\right)\right)$. Therefore

$$
\sum_{\nu=1}^{u} p^{\prime}(\underline{w}, \nu)=p_{S_{\{r\}}}^{\prime}(\underline{w})-p_{S_{\{r\}}}^{\prime}\left(\underline{w}-\xi^{(r)}\right)=A(\underline{w}),
$$

as desired.

Corollary 5.2. More generally, if $A \subseteq I$, then we have

$$
\left.P_{S}(\underline{t})\right|_{\left\{t_{i}=1 \mid i \notin A\right\}}=\prod_{j \notin A}\left(1-\underline{t}_{A}^{\xi_{j}}\right) P_{S_{A}}\left(\underline{t}_{A}\right),
$$

where ${\underline{\xi_{A}}}_{A}^{\xi_{A}}:=\prod_{i \in A} t_{i}^{\xi_{i, j}}$ and $P_{S_{A}}\left(\underline{t}_{A}\right)$ is the Poincaré polynomial of $S_{A}$.

Next result seems to involve non-trivial computations on $P$ :

Theorem 5.3. The intersection multiplicities $\xi_{i, j}$ between couples of branches of $\mathcal{O}$ can be computed from $P_{S}$.

Proof. First, we may apply recursively the projection formula to obtain

$$
P_{S}^{\prime}(1, \ldots 1, t, 1, \ldots, 1)=\prod_{\substack{j=1 \\ i}}^{r}\left(1-\underline{t}^{\xi_{i, j}}\right) \cdot P_{S_{\{i\}}}^{\prime}(t) .
$$

At this point, we need a theorem of Campillo, Delgado and GuseinZade (cf. [4; also [17]) which expresses the Poincaré series of an irreducible plane curve singularity in terms of a minimal set of generators of the corresponding value semigroup. More precisely, assuming the set $\left\{\rho_{0}^{i}, \rho_{1}^{i}, \ldots, \rho_{g}^{i}\right\}$ to be a minimal set of generators of $S_{\{i\}}$, and writing $\theta_{0}^{i}:=\rho_{0}^{i}, \theta_{k+1}^{i}:=\operatorname{gcd}\left(\rho_{0}^{i}, \ldots, \rho_{k}^{i}\right)$ for $k \in\{1, \ldots, g\}$, and $N_{k}^{i}=\frac{\theta_{k}^{i}}{\theta_{k+1}^{i}}$ for all $k \in\{1, \ldots, g\}$ and all $i \in\{1, \ldots, r\}$, we have

$$
P_{S_{\{i\}}}^{\prime}(t)=(t-1) \sum_{n \in S_{\{i\}}} t^{n}=(t-1) \cdot \frac{1}{1-t^{\rho_{0}^{i}}} \cdot \prod_{k=1}^{g} \frac{\left(1-t^{N_{k}^{i} \rho_{k}^{i}}\right)}{\left(1-t^{\rho_{k}^{i}}\right)}
$$


is a polynomial non divisible by $t-1$ (see e. g. [5]), and because of the fact that $(t-1) \sum_{i=0}^{m-1} t^{i}=\left(t^{m}-1\right)$, it is also not divisible by $t^{m}-1$ for any natural number $m$. Therefore we can write

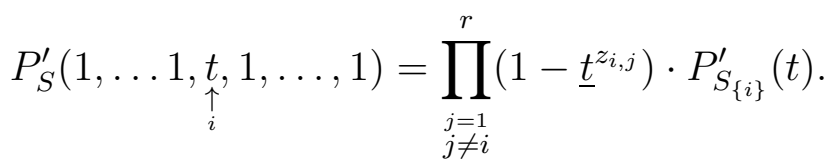

with $z_{i, j} \in \mathbb{N}$, and this factorisation is uniquely determined. By comparing now the factors $\prod_{\substack{j=1 \\ j \neq i}}^{r}\left(1-\underline{t}^{z_{i, j}}\right)$ and $\prod_{\substack{j=1 \\ j \neq i}}^{r}\left(1-\underline{t}^{\xi_{i, j}}\right)$ for every $i \in\{1, \ldots, r\}$, we obtain the following system of linear equations in the indeterminates $z_{i, j}$ :

$$
\left.\begin{array}{rl}
z_{1,2}+z_{1,3}+z_{1,4}+\ldots+z_{1, r} & =\xi_{1,2}+\xi_{1,3}+\xi_{1,4}+\ldots+\xi_{1, r} \\
z_{2,1}+z_{2,3}+z_{2,4}+\ldots+z_{2, r} & =\xi_{2,1}+\xi_{2,3}+\xi_{2,4}+\ldots+\xi_{2, r} \\
z_{3,1}+z_{3,2}+z_{3,4}+\ldots+z_{3, r} & =\xi_{3,1}+\xi_{3,2}+\xi_{3,4}+\ldots+\xi_{3, r} \\
& \vdots \\
z_{r, 1}+z_{r, 2}+z_{r, 3}+\ldots+z_{r, r-1} & =\xi_{r, 1}+\xi_{r, 2}+\xi_{r, 3}+\ldots+\xi_{r, r-1}
\end{array}\right\}
$$

The resolution of the system yields the desired result.

Remark 5.4. As a corollary from the previous results one has that the polynomials $P_{S_{A}}$, for $A \subseteq I$, can be computed from $P_{S}$. This provides - for complex plane curve singularities - an alternative way to prove that the Alexander polynomial is equivalent to the topological type of the singularity, and it was already proved with topological methods in [8]. In particular, they describe how to reconstruct the resolution tree from the Poincaré series. It is worth pointing out that Yamamoto was the first who proved that the Alexander polynomial determines the topology of an algebraic link by topological methods ([26]). Also, it provides a proof that $P_{S}^{\prime}$ and $\widetilde{P}_{S}$ are equivalent data.

\section{Generalised Poincaré series}

One can now pose the question Section 5 deals with in the context of Poincaré series of motivic nature (called generalised Poincaré series following the terminology introduced in [7]). Let us present first the basic notions.

Let $K_{0}\left(\boldsymbol{\nu}_{k}\right)$ be the Grothendieck ring of quasi-projective varieties. It is generated by classes $[X]$ of such varieties subject to the relations:

i) if $X_{1} \cong X_{2}$ then $\left[X_{1}\right]=\left[X_{2}\right]$;

ii) if $Y$ is Zariski-closed in $X$, then $[X]=[Y]+[X \backslash Y]$ 
(the multiplication is defined by the Cartesian product). Let $\mathbb{L}$ be the class $\left[\mathbb{A}_{k}^{1}\right]$ of the complex affine line. The class $\mathbb{L}$ is not equal to zero in the ring $K_{0}\left(\boldsymbol{\nu}_{k}\right)$. Moreover the natural ring homomorphism $\mathbb{Z}[X] \rightarrow K_{0}\left(\boldsymbol{\nu}_{k}\right)$ which sends $X$ to $\mathbb{L}$ is an inclusion. Let $K_{0}\left(\boldsymbol{\nu}_{k}\right)_{(\mathbb{L})}$ be the localisation of the Grothendieck ring $K_{0}\left(\boldsymbol{\nu}_{k}\right)$ by the class $\mathbb{L}$. The natural homomorphism $\mathbb{Z}[X]_{(X)} \rightarrow K_{0}\left(\nu_{k}\right)_{(\mathbb{L})}$ is an inclusion as well.

The generalised Euler characteristic $\chi_{g}(X)$ of a cylindric subset $X$ is the element $[Y] \cdot \mathbb{L}^{-d(p)}$ in the ring $K_{0}\left(\boldsymbol{\nu}_{k}\right)_{(\mathbb{L})}$, where $Y=\pi_{p}^{-1}(X)$ is a constructible subset of $\mathbb{P O}$, as in the previous subsection. Note that $\chi_{g}(X)$ is well-defined, because if $X=\pi_{q}^{-1}\left(Y^{\prime}\right), Y^{\prime} \subset \mathbb{P} J_{V, 0}^{q}$ and $p \geq q$, then $Y$ is a locally trivial fibration over $Y^{\prime}$ with the fibre $k^{d(p)-d(q)}$ and therefore $[Y]=\left[Y^{\prime}\right] \cdot \mathbb{L}^{d(p)-d(q)}$.

Let us take the usual Euler characteristic $\chi(\cdot)$. Let $A$ (resp. $A^{\prime}$ ) be a subspace of $\mathcal{O}$ of finite codimension $a$ (resp. $a^{\prime}$ ) with $a^{\prime}>a$. Let be $Q:=\mathbb{L}^{-1}$. Then one has

$$
\begin{aligned}
\operatorname{dim}_{k}\left(A / A^{\prime}\right) & =\chi\left(\mathbb{P} A \backslash \mathbb{P} A^{\prime}\right) \\
& =\chi\left(\mathbb{P}\left(A / A^{\prime}\right)\right) .
\end{aligned}
$$

These equalities do not hold for the generalised Euler characteristic. Nevertheless we can take Equation (5) as definition of a sort of "generalised (or motivic) dimension" just by taking (5) with $\chi_{g}(\cdot)$ instead of the usual $\chi(\cdot)$. Hence using the cellular decomposition of a projective space one gets

$$
\begin{aligned}
\chi_{g}\left(\mathbb{P} A \backslash \mathbb{P} A^{\prime}\right) & =Q^{a}+Q^{a+1}+\ldots+Q^{a^{\prime}-1} \\
& =Q^{a+1} \cdot \frac{1-Q^{a^{\prime}-a}}{1-Q} .
\end{aligned}
$$

If we set $\ell(\underline{v}):=\operatorname{dim}_{k}(\mathcal{O} / J(\underline{v}))$ and put $A=J(\underline{v}), A^{\prime}=J(\underline{v}+1)$, $a=\ell(\underline{v})$ and $a^{\prime}=\ell(\underline{v}+1)$ in Equation (17), then we define a series

$$
L_{g}(\underline{t}):=\sum_{\underline{v} \in \mathbb{Z}^{r}} Q^{\ell(\underline{v})+1} \cdot \frac{1-Q^{\ell(\underline{v}+1)-\ell(\underline{v})}}{1-Q} \cdot \underline{t}^{\underline{v}}
$$

which is a "motivic version" of the series $L(\underline{t})$ given by the formula (2). One can also see (cf. [7, Proposition 2]):

$$
P_{g}(\underline{t})=\frac{L_{g}(\underline{t}) \cdot \prod_{i=1}^{r}\left(t_{i}-1\right)}{t_{1} \cdot \ldots \cdot t_{r}-1} .
$$

On the other hand, the equality between Equations (5) and (6) does not hold when we replace $\chi(\cdot)$ with $\chi_{g}(\cdot)$ :

$$
\left.\chi_{g}\left(\mathbb{P} A \backslash \mathbb{P} A^{\prime}\right)\right) \neq \chi_{g}\left(\mathbb{P}\left(A / A^{\prime}\right)\right) .
$$


For $A=J(\underline{v})$ and $A^{\prime}=J(\underline{v}+1)$ we get

$$
\chi_{g}(\mathbb{P}(J(\underline{v}) / J(\underline{v}+1)))=\frac{Q^{\ell(\underline{v})+1}}{Q^{\ell(\underline{v}+\underline{1})}} \cdot \frac{1-Q^{\ell(\underline{v}+\underline{1})-\ell(\underline{v})}}{1-Q},
$$

therefore we define the corresponding series as

$$
\widehat{L}_{g}(\underline{t}):=\sum_{\underline{v} \in \mathbb{Z}^{r}} \frac{Q^{\ell(\underline{v})+1}}{Q^{\ell(\underline{v}+\underline{1})}} \cdot \frac{1-Q^{\ell(\underline{v}+\underline{1})-\ell(\underline{v})}}{1-Q} \underline{t}^{\underline{v}} .
$$

Lemma 6.1.

$$
\widehat{L}_{g}(\underline{t})=\sum_{\underline{v} \in \mathbb{Z}^{r}}[\mathbb{P}(J(\underline{v}) / J(\underline{v}+\underline{1}))] \underline{t} \underline{v} .
$$

Proof. The class in $K_{0}\left(\boldsymbol{\nu}_{k}\right)$ of the projectivisation of the vector space $J(\underline{v}) / J(\underline{v}+\underline{1})$ (of finite dimension) is equal to $(\mathbb{L}-1)^{-1}\left(\mathbb{L}^{c(\underline{v})}-1\right)$. By setting $Q:=\mathbb{L}^{-1}$ this is equal to $(1-Q)^{-1} Q\left(Q^{\ell(\underline{v})-\ell(\underline{v}+\underline{1})}-1\right)$, and this coincides with the coefficient of the definition of $\widehat{L}_{g}(\underline{t})$.

The following result shows a "motivic" series analogous to the series resulting from taking Euler characteristic to the spaces $\mathbb{P} F_{\underline{v}}$ of the formula (1):

\section{Proposition 6.2.}

$$
\widehat{P}_{g}(\underline{t})=\frac{\widehat{L}_{g}(\underline{t}) \cdot \prod_{i=1}^{r}\left(t_{i}-1\right)}{t_{1} \cdot \ldots \cdot t_{r}-1} .
$$

Notice that the series $\widehat{P}_{g}(\underline{t})$ coincides with $P(\underline{t})$ in the case $r=1$.

As a difference from the classical Poincaré polynomial, the generalised Poincaré series $P_{g}(\underline{t})$ does not satisfy a property analogous to that of Theorem 5.1, but it just forget components, as E. Gorsky has shown in [15] for the complex case. Let us define

$$
\bar{P}_{g}\left(t_{1}, \ldots, t_{r}\right):=\left(1-Q t_{1}\right) \cdot \ldots \cdot\left(1-Q t_{r}\right) \cdot P_{g}\left(t_{1}, \ldots, t_{r}\right) .
$$

Moreover, consider the series $P_{g}^{S_{\{r\}}}\left(t_{1}, \ldots, t_{r-1}\right)$ be the generalisd Poincaré series associated with the ring consisting of the branches $C_{1}, \ldots, C_{r-1}$ corresponding to the local rings $V_{1}, \ldots, V_{r-1}$, and

$$
\bar{P}_{g}^{S_{\{r\}}}\left(t_{1}, \ldots, t_{r-1}\right):=\left(1-Q t_{1}\right) \cdot \ldots \cdot\left(1-Q t_{r-1}\right) \cdot P_{g}^{S_{\{r\}}}\left(t_{1}, \ldots, t_{r-1}\right)
$$

Proposition 6.3 (Gorski). For $\mathcal{O}$ reduced with $r>1$ branches we have

$$
\bar{P}_{g}\left(t_{1}, \ldots, t_{r-1}, t_{r}=1\right)=(1-Q) \bar{P}_{g}^{S_{\{r\}}}\left(t_{1}, \ldots, t_{r-1}\right) .
$$

If $\mathcal{O}$ is irreducible, then $\bar{P}_{g}(t=1)=1$. 
It implies in particular that these generalised Poincaré series are not affected by "forgetting components", as a difference with the non-motivic Poincaré polynomial (cf. Theorem 5.1).

As we did in the non-motivic case, one can also define a motivic version of the Poincaré series $H(\underline{t})$, namely

$$
H_{g}(\underline{t}):=\sum_{\underline{v} \in \mathbb{Z}_{\geq 0}^{r}}[\mathcal{O} / J(\underline{v})] \underline{\underline{v}} .
$$

Similarly as done for the series $H(\underline{t})$ in Corollary 4.3 we may also prove the following result:

\section{Proposition 6.4.}

$$
\prod_{i=1}^{r}\left(1-t_{i}\right) H_{g}(\underline{t})=\sum_{\varnothing \neq A \subseteq I}(-1)^{\sharp(A)-1} \underline{t}_{A} P_{g_{S_{A}}}\left(\underline{t}_{A}\right) .
$$

\section{ACKNOWLEDGEMENTS}

The author wishes to express his gratitude to Prof. Dr. Félix Delgado de la Mata for stimulating conversations and helpful remarks about the topic.

\section{REFERENCES}

[1] A. Campillo, F. Delgado, S.M. Gusein-Zade: On the generators of the semigroup of a plane curve singularity. J. London Math. Soc. (2) 60, 149-167 (1999)

[2] A. Campillo, F. Delgado, S.M. Gusein-Zade: The extended semigroup of a curve singularity. Proc. of the Steklov Institute of Math. 221 (in Honour of V.I. Arnold) (1998), pp. 139-156 (1998)

[3] A. Campillo, F. Delgado, S.M. Gusein-Zade: On the monodromy of a plane curve singularity and the Poincaré series of its ring of functions. Functional Analysis and its Applications 33 (1), 56-57 (1999)

[4] A. Campillo, F. Delgado, S.M. Gusein-Zade: On the monodromy at infinity of a plane curve and the Poincaré series of its coordinate ring. Topology 8 (In Honour of Pontryagin, Moscow, 1998). English version: J. Math. Sci. 105 (2), 1839-1842 (2001)

[5] A. Campillo, F. Delgado, S.M. Gusein-Zade: The Alexander polynomial of a plane curve singularity via the ring of functions on it. Duke Math. J. 117 (1), 125-156 (2003)

[6] A. Campillo, F. Delgado, S.M. Gusein-Zade: The Alexander polynomial of a plane curve singularity and integrals with respect to the Euler characteristic. Int. J. of Math. 14 (1), 47-54 (2003)

[7] A. Campillo, F. Delgado, S.M. Gusein-Zade: Multi-index filtrations and motivic Poincaré series. Monatshefte für Mathematik 150, 193-209 (2007)

[8] A. Campillo, F. Delgado, S.M. Gusein-Zade: The Poincaré series of divisorial valuations in the plane defines the topology of the set of divisors. Funct. Anal. Other Math. 3, 39-46 (2010), DOI 10.1007/s11853-010-0040-9. 
[9] A. Campillo, F. Delgado, S.M. Gusein-Zade, F. Hernando: Poincaré series of collections of plane valuations. Int. J. of Math. 21 (11), 1461-1473 (2010), DOI 10.1142/S0129167X10006586

[10] A. Campillo, F. Delgado, K. Kiyek: Gorenstein property and symmetry for one-dimensional local Cohen Macaulay rings. Manuscripta Math. 83 (1994), 405-423 (1994)

[11] F. Delgado: The semigroup of values of a curve singularity with several branches. Manuscripta Math. 59 (1987), 347-374 (1987)

[12] F. Delgado, C. Galindo, A. Núñez: Generating sequences and Poincaré series for a finite set of plane divisorial valuations. Advances in Math. 219(5), 16321655 (2008)

[13] F. Delgado, J.J. Moyano-Fernández: On the relation between the generalized Poincaré series and the Stöhr zeta function. Proc. Am. Soc. 137(1), 5159 (2009)

[14] V.M. Galkin: Zeta functions of some one-dimensional rings. Izv. Akad. Nauk. SSSR Ser. Mat 37, 3-19 (1973)

[15] E. Gorsky: Combinatorial computation of the motivic Poincaré series. Journal of Singularities 3, 48-82 (2011)

[16] B. Green: Functional equations for zeta functions of non-Gorenstein orders in global fields. Manuscripta mathematica 64, 485-502 (1989)

[17] K. Kiyek, J.J. Moyano-Fernández: The Poincaré series of a simple complete ideal of a two-dimensional regular local ring. Journal of Pure and Applied Algebra 213, 1777-1787 (2009)

[18] K. Kiyek, J.L. Vicente: Resolution of Curve and Surface Singularities in Characteristic Zero. Kluwer, Dordrecht, 2004.

[19] J.J. Moyano-Fernández: Generalised Poincaré series and embedded resolution of curves. Monatshefte für Math., on-line first, DOI:10.1007/s00605-010-0259-z

[20] J.J. Moyano-Fernández, W.A. Zúñiga: Motivic zeta functions for curve singularities. Nagoya Math. Journal 198, 47-75 (2010)

[21] J.J. Moyano-Fernández: Fractional ideals and integration with respect to the generalised Euler characteristic. Preprint, ArXiv XXXXXX (2011)

[22] K.O. Stöhr: Local and Global Zeta-Functions of Singular Algebraic Curves. Journal of Number Theory 71, 172-202 (1998)

[23] K.O. Stöhr: Multi-variable Poincaré Series of Algebraic Curve Singularities over Finite Fields. Mathematische Zeitschrift 262(4), 849-866 (2009)

[24] G. Torres: On the Alexander polynomial. Ann. of Math. 57(1), 57-89 (1953)

[25] R. Waldi: Wertehalbgruppe und Singularität einer ebenen algebraischen Kurve. Dissertation. Regensburg (1972)

[26] M. Yamamoto: Classification of isolated algebraic singularities by their Alexander polynomials. Topology 23, 227-287 (1984)

Institut für Mathematik, Universität Osnabrück. Albrechtstrasse 28A, D-49076 Osnabrück, Germany

E-mail address: jmoyanof@uni-osnabrueck.de 